\title{
Sur les notions d'usage chez Wittgenstein et Heidegger
}

\author{
Frédérique LAURENT (Paris , 1.frederique@ noos.fr), François-Igor PRIS (Dortmund, \\ frigpr@gmail.com)
}

\section{Résumé}

Nous comparons les notions d'usage et de signification chez Ludwig Wittgenstein et Martin Heidegger. Contrairement à Jocelyn Benoist, nous pensons que l'analogie entre Wittgenstein et Heidegger n'est pas superficielle. La métaphysique de Heidegger explicite certaines présuppositions implicites de la seconde philosophie de Wittgenstein. Le pragmatisme naturaliste de Wittgenstein peut être théorisé. Notamment la notion wittgensteinienne d'usage, ou de jeu de langage, peut être comprise comme une pratique à la fois naturelle et normative régie par des règles.

Mots clés : usage, signification, comprendre, jeu de langage, Dasein, signe, instrument/outil, aspect, voir-comme, Wittgenstein, Heidegger.

\section{Abstract}

Wittgenstein's notions of use and meaning are compared with the corresponding Heidegger's ones. Unlike Jocelyn Benoist, we suggest that the analogy between Wittgenstein and Heidegger is not superficial. Heidegger's metaphysics makes explicit some of the implicit presuppositions of Wittgenstein's philosophy. Wittgenstein's naturalistic pragmatism can be theorized. In particular, Wittgenstein's notion of use, or language game, can be understood as both natural and normative rulegoverning practice.

Key words : use, meaning, understanding, language game, Dasein, sign, instrument/tool, aspect, seeing-as, Wittgenstein, Heidegger

1. Dans le $\S 43$ des Investigations philosophiques, Wittgenstein écrit: « Die Bedeutung eines Wortes ist sein Gebrauch in der Sprache. » («La signification d'un mot est son usage dans le langage.» Notre traduction.) Cela concerne justement aussi la signification du mot « signification ».

Mais qu'est ce que c'est, l' « usage », et comment faut-il comprendre « est » ? Ne serait-il pas plus correct de dire que la signification d'un mot est associée à son usage ? Peut-on introduire une ontologie des usages fondant les significations?

Ces questions peuvent être formulées en d'autres termes. Une lecture attentive de Wittgenstein montre que les termes «usage » et «jeu de langage » sont synonymiques. Par 
exemple, le mot est employé au sein d'un jeu de langage. Le jeu de langage est l'usage d'une règle qui le régit. Le mot, ou le concept correspondant, fournit un exemple de la règle.

Le signe est un autre exemple de la règle. Par ailleurs, il n'existe pas en tant que tel, mais seulement en relation avec ses usages; il n'est pas séparable de ceux-ci. L'usage du signe s'identifie au symbole dans lequel ce premier se « dissout », devient implicite.

Une théorisation de la philosophie de Wittgenstein en termes de pratiques normatives naturelles - jeux de langage et formes de vie (c'est-à-dire ensemble des jeux de langage) la rapproche du pragmatisme normatif de Heidegger ${ }^{1}$. (Wittgenstein et Heidegger sont eux aussi naturalistes ${ }^{2}$.) Heidegger, quant à lui, assigne un rôle fondamental à la notion d' « usage ».

Néanmoins, Jocelyn Benoist conteste le caractère profond de l'analogie entre Wittgenstein et Heidegger ${ }^{3}$.

Nous revenons ici à la question de cette analogie. Nous essayons également de déceler si une théorisation quelconque de la notion wittgensteinienne d'usage est effectivement possible.

2. Si la signification d'un mot est son usage, alors comprendre l'un, c'est comprendre l'autre. Dans un sens, nous comprenons la signification d'un mot si nous l'employons ou sommes capables de l'employer.

Wittgenstein fait une distinction entre deux notions d'usage et donc de signification. D'un coté, il peut s'agir d'une ambigüité lexicale, c'est-à-dire d'usages de différents concepts ; de l'autre - de différents usages d'un seul et même concept.

Par exemple, un mot peut être ambigu ou ses usages peuvent être très différents (correspondant aux concepts différents). Tels usages correspondent aux différentes significations d'un mot isolé ou à celles de différents concepts.

Par exemple, dans le $\S 282$ des Investigations philosophiques Wittgenstein écrit ${ }^{4}$ : «Ja; wir sagen von Leblosem, es habe Schmerzen: im Spiel mit Puppen z.B. Aber diese Verwendung des Schmerzbegriffs ist eine sekundäre.» («En effet, nous disons d'une chose inanimée qu'elle souffre: en jouant avec des poupeés: par exemple. Mais cet emploi du concept de douleur en est un secondaire.»)

Un mot (un concept), ayant une signification fixe, peut être employé dans des contextes différents ${ }^{5}$. Ces usages révèlent des aspects différents de sa signification. «Ich identifiziere

\footnotetext{
${ }^{1}$ Brandom Robert, Tales of the Mighty Dead, Cambridge: Harvard, 2002.

${ }^{2}$ Rouse Joseph, How Scientific Practices Matter, Chicago: Chicago, 2002.

${ }^{3}$ Benoist Jocelyn, « Le mythe de l'usage », Les Etudes philosophiques, 2010, № 4 (3), pp. 417-432.

${ }^{4}$ Wittgenstein Ludwig, Philosophical Investigations (1953), Blackwell Publishing, 2001, § 282. Wittgenstein Ludwig, Tractatus logico-philosophiques suivi d'investigations philosophiques, tr. fr. Pierre Klossowski, Gallimard, 1961, § 282.

${ }^{5}$ Selon Heidegger, «Der Begriff des Sinnes umfaßt das formale Gerüst dessen, was notwendig zu dem gehört, was verstehende Auslegung artikuliert. » (Le concept de sens embrasse la structure formelle de ce qui appartient nécessairement à ce que l'explicitation compréhensive articule.) (Heidegger Martin, Sein und Zeit, Tübingen, Max Niemeyer, 1967 (accessible sur Internet), 2006, p. 151. Heidegger Martin, Etre et temps, tr. fr. Emmanuel Martineau, Edition numérique Hors-Commerce, 1985, p. 132.) Nous interprétons cette «structure formelle» comme la signification conceptuelle. C'est la signification d'un mot (d'une phrase) isolé ou celle du concept
} 
meine Empfindung freilich nicht durch Kriterien, sondern ich gebrauche den gleichen Ausdruck.» («Bien entendu, je n'identifie pas ma sensation par des critères: mais j'use de la même expression $\left.{ }^{6} . »\right)$

Chez Heidegger aussi, on peut distinguer deux notions d'usage et, respectivement, de la signification - qui est l'existential du Dasein (Sinn ist ein Existenzial des Daseins ${ }^{7}$ ) - et du comprendre.

D'un coté, pour Heidegger comprendre c'est être capable de faire quelque chose. «Wir gebrauchen zuweilen in ontischer Rede den Ausdruck „etwas verstehen“ in der Bedeutung von „einer Sache verstehen können“, „ihr gewachsen sein“, „etwas können“8.» («Dans un langage ontique, nous prenons parfois l'expression „comprendre quelque chose“ au sens de : „s'entendre à quelque chose“, c'est-à-dire ,pouvoir y faire face“, „savoir se tirer d'affaire“" 9 . »)

Cette sorte du comprendre (respectivement, de la signification) correspond à la capacité d'employer la règle/le concept et donc mutatis mutandis à la notion wittgensteinienne d'usage dans le premier sens.

Heidegger écrit également: «Verstehen ist das existenziale Sein des eigenen Seinkönnens des Daseins selbst, so zwar, daß dieses Sein an ihm selbst das Woran des mit ihm selbst Seins erschließ $\mathrm{t}^{10}$.» («Le comprendre est l'être existential du pouvoir-être propre du Dasein luimême, de telle sorte que cet être ouvre en lui-même „où“ “il en est avec lui-même ${ }^{11}$. »)

Cette sorte du comprendre (et de la signification) correspond au comprendre de l'usage d'une règle et donc la notion wittgensteinienne d'usage au second sens. ${ }^{12}$

On peut donc être d'accord avec Jocelyn Benoist dans la mesure où, pour Heidegger aussi, la signification est l'usage. ${ }^{13}$

La différence entre les deux philosophes dans leur vision de la signification comme l'usage réside en ce que Wittgenstein propose une analyse thérapeutique de la notion de la signification en termes d'usage, tandis que Heidegger considère l'usage comme une condition métaphysique de la signification comme telle.

(règle) correspondant. La signification complète est pragmatique ; c'est la signification de l'usage. (Voir aussi ci-dessous.)

${ }^{6}$ Ibidem, $§ 290$.

${ }^{7}$ Heidegger Martin, Sein und Zeit (1926), Tübingen, Max Niemeyer Verlag, 1967 (accessible sur Internet), 2006, p. 151.

${ }^{8}$ Ibidem, $\S 31$, p. 143.

${ }^{9}$ Heidegger Martin, Être et temps, tr. fr. Emmanuel Martineau, Edition numérique Hors-Commerce, 1985, p. 127.

${ }^{10}$ Heidegger Martin, Sein und Zeit, § 31, p. 144.

${ }^{11}$ Heidegger Martin, Être et temps, p. 127.

${ }^{12}$ Il existe une relation évidente entre les deux notions d'usage. La règle comme l'usage (d'une certaine superrègle) est tout simplement un usage plus général (par rapport à l'usage de cette règle). Donc pour l'essentiel la notion d'usage, ainsi que celle de la signification, est une.

${ }^{13}$ Benoist Jocelyn, « Le mythe de l'usage », Les Etudes philosophiques, 2010, № 4 (3), pp. 417-432. 
Wittgenstein parle plus de l'usage du langage ; Heidegger - de l'usage du monde lui-même (pour lui, la signification primaire c'est la «signification» de l'usage du monde), par exemple, d'un marteau ou d'une poignée de porte. Bien entendu, la pratique matérielle est différente de celle purement symbolique ou linguistique. Néanmoins, celle-ci peut être mélangée à celle-là (chez Wittgenstein il y a beaucoup d'exemples de jeux de langage contenant des éléments proprement linguistiques ainsi que ceux du monde matériel. Voir notamment son exemple de l'achat de cinq pommes rouges $(\S 1)$ ou son exemple avec des matériaux de construction ( $(2)$ au début même des Investigations Philosophiques) ou connectée à elle indirectement. Cela dit, pour ces deux philosophes le langage correctement employé est dans une relation intime avec le monde. ${ }^{14}$ Cela diminue la différence entre eux. ${ }^{15}$

Dans le cadre de l'approche wittgensteinienne contextualiste de Jocelyn Benoist ${ }^{16}$ la condition de l'existence d'une relation intime entre le langage et le monde est celle de l'adéquation. Celle-ci signifie ${ }^{17}$ :

«(...) that the concept, or whatever represents what is given might find its perfect match in the given, that the gap that there seems to be between the concepts and the individuals that serve as their examples in the traditional conception might be closed, and that real pieces of givenness might really correspond to the characters included in the representation.» («Que le concept ou quoi que ce soit qui représente ce qui est donné, pourrait trouver sa correspondance parfaite avec le donné, que le fossé qui semble exister entre les concepts et les individuals qui servent en tant que leurs exemples dans le cadre de la conception traditionnelle, pourrait être fermé, et que les parties réelles du donné pourraient vraiment correspondre aux caractères inclus dans la représentation.» Notre traduction.)

À son tour Heidegger écrit ${ }^{18}$ : «Aussage ist kein freischwebendes Verhalten, das von sich aus primär Seiendes überhaupt erschließen könnte, sondern hält sich schon immer auf der Basis des In-der-Welt-seins. » («L'énoncé n'est pas un comportement flottant en l'air qui pourrait de lui-même et primairement ouvrir de l'étant en général, mais il se tient toujours déjà sur la base de l'être-au-monde.»)

Peut-on établir une relation plus étroite entre Wittgenstein et Heidegger? Peut-on dire que Heidegger explicite les présupposés métaphysiques qui sont implicites dans la philosophie de Wittgenstein?

3. Les deux philosophes, Wittgenstein et Heidegger, ont recours à la métaphore de l'instrument/outil, qui n'est pas chez eux simplement une métaphore.

\footnotetext{
${ }^{14}$ On peut employer un marteau d'une façon ou d'une autre, et on peut parler de son usage. Dans le premier cas on a directement affaire avec le monde lui-même, tandis que dans le second - avec le langage. Cela dit pour Wittgenstein ainsi que pour Heidegger le «fossé » épistémique entre le langage et le monde se dissout si l'on emploi le langage correctement.

${ }^{15}$ Notons que Heidegger (ainsi que Wittgenstein) parle aussi de l'usage des signes qui, chez lui, jouent le rôle d'instruments (voir, par exemple, Sein und Zeit, § 17. Voir aussi $§ 4$ ci-dessous. Sur la notion d'instrument chez Wittgenstein et Heidegger voir $\S 3$ ci-dessous). À son tour Wittgenstein considère non seulement des signes linguistiques mais aussi matériels, par exemple, tels que les signes routiers - l'exemple qu'on trouve aussi chez Heidegger.

${ }^{16}$ Benoist Jocelyn, Concepts, Paris, Les éditions CERF, 2010/2011. Benoist Jocelyn, Eléments de philosophie réaliste, Paris, Vrin, 2011. Benoist Jocelyn, « Making Ontology Sensitive », Cont. Philos. Rev., 2012, 45, pp. 411-424.

${ }^{17}$ Benoist Jocelyn, « Making Ontology Sensitive », p. 412.

${ }^{18}$ Heidegger Martin, Sein und Zeit, p. 156. Heidegger Martin, Etre et temps, p. 136.
} 
Chez Wittgenstein, comme nous le comprenons, la notion d'instrument au sens large coïncide avec celle de la règle. L'usage est l'usage d'un «instrument», c'est-à-dire d'une règle. Autrement dit, l'usage est le jeu de langage.

Wittgenstein lui-même, dans l'esprit de son style anti-théorique, n'introduit pas une ontologie des usages. Il ne pose pas explicitement la question sur la nature des usages, ou jeux de langage. Mais peut-on le faire en principe ? Le Dasein heideggérien n'est-il pas, par exemple, une telle ontologisation de la notion d'usage ou celle de jeu de langage ${ }^{19}$ ?

Si nous prenons un instrument au sens le plus ordinaire de ce mot, par exemple, un marteau l'exemple analysé par les deux philosophes, ce qu'il faut entendre par ses usages est clair. En outre, ce qu'il faut entendre par ses usages non-standards (un marteau peut être utilisé pour planter un clou mais aussi comme une arme) est clair.

Wittgenstein analyse la notion d'usage d'un marteau à l'aide de sa notion d'air de famille (voir notre interprétation ci-dessous), et il établit une analogie entre l'usage d'un marteau et l'usage d'un mot (cette analogie se transforme en identité, si l'on passe d'un usage d'un marteau à une description adéquate de son usage dans le langage).

«Do we use a hammer in two different ways when we hit a nail with it and, on the other hand, drive a peg into a hole? And do we use it in two different ways or in the same way when we drive this peg into this hole and, on the other hand, another peg into another hole? Or should we only call it different uses when in one case we drive something into something and in the other, say, we smash something? Or is this all using the hammer in one way and is it to be called a different way only when we use the hammer as a paper weight? - In which cases are we to say that a word is used in two different ways and in which that it is used in one way? To say that a word is used in two (or more) different ways does in itself not yet give us any idea about its use. It only specifies a way of looking at this usage by providing a schema for its description with two (or more) subdivisions. It is all right to say: "I do two things with this hammer; I drive a nail into this board and one into that board". But I could also have said: "I am doing only one thing with this hammer; I am driving a nail into this board and one into that $\operatorname{board}^{20} . »$

Nous interprétons les mots de Wittgenstein en termes d'air de famille du premier et du second ordre (notre terminologie). L'air de famille du premier ordre est celle entre les usages d'un seul et même concept (règle, ou « instrument » au sens large). C'est ce à quoi dans le $\S 2$ nous avons fait référence en parlant de l'usage dans le second sens. L'air de famille du second ordre est celui entre les usages d'un certain super-concept. C'est-à-dire qu'il s'agit d'une ressemblance entre les concepts, ou entre les usages d'un concept et sa généralisation (qui est une ressemblance plus éloignée que celle du premier ordre). C'est ce à quoi nous avons fait référence ci-dessus en parlant de l'usage dans le premier sens.

L'air de famille peut être violé dans le sens où l'on peut former deux (sous)-concepts à partir du concept correspondant.

Le choix d'un concept (d'un point de vue, d'une classification) lui-même dépend d'un contexte, ou d'une perspective. Mais le contexte d'usage (d'un concept ou super-concept)

\footnotetext{
${ }^{19}$ Une «forme de vie» comme un ensemble de jeux de langage peut être considérée comme un jeu de langage d'un ordre supérieur. Donc la question posée est également celle de savoir si la notion heideggérienne du Dasein correspond à celle wittgensteinienne de la forme de vie. John Haugeland, par exemple, écrit: «Dasein is neither people nor their being, but rather a way of life shared by the members of some community.» («Dasein n'est ni des gens ni leur étant, mais plutôt une façon de vivre partagée par les membres d'une communauté donnée.» Notre traduction.) (Haugeland John, «Reading Brandom Reading Heidegger», European Journal of Philosophy, 2005, 13:3, pp. 421-428, p. 423.)
}

${ }^{20}$ Wittgenstein Ludwig, The Blue Book, Oxford, Blackwell, 1958, p. 58. 
n'est pas séparé de l'usage ; il est une partie inséparable de l'usage même. Pour cette raison l'usage, ou le jeu de langage, n'est pas substantiel dans le sens où il a une dimension normative. Il peut être correct (usage d'une règle) ou non. Il est donc justifiable.

En reprenant l'exemple wittgensteinien avec le marteau, la ressemblance entre planter un clou et planter un autre clou est plus forte que celle entre planter un clou et planter un piquet, bien que dans un contexte défini chaque ressemblance puisse être violée (et inversement, dans un contexte des usages qui ne se ressemblent pas peuvent être considérés comme similaires).

Heidegger analyse la notion de marteau à l'aide de sa notion du Dasein. L'usage d'un marteau est (l'usage) du Dasein.

Cela dit, un instrument peut être employé de façon plus ou moins réflexive, plus ou moins automatique, sans être purement mécanique, mais toujours consciemment. Dasein est le cas limite de l'usage automatique d'un instrument, quand le sujet n'est conscient ni de soi-même ni de l'instrument; dans ce cas-là, la conscience est une pure expérience phénoménale de l'usage d'un instrument. «(...) Je weniger das Hammerding nur begafft wird, je zugreifender es gebraucht wird $(\ldots)^{21} . »(\ll(\ldots)$ Moins la chose-marteau est simplement ,regardée“, plus elle est utilisée efficacement $(\ldots)^{22}$.») Heidegger oppose l'usage à la contemplation théorique. En même temps, pour lui, l'usage d'un instrument (d'une chose) n'est pas « aveugle »; il a sa propre « manière de voir » (Sichtart).

D'une façon analogue, l'usage wittgensteinien d'une règle, c'est-à-dire un jeu de langage, peut être plus ou moins réflexif. Le jeu de langage «instinctif » (le suivre «aveugle » de la règle à la Wittgenstein) a sa propre phénoménologie, sa propre façon de voir. On peut dire de lui la même chose que Wittgenstein dit de la sensation: «Sie ist kein Etwas, aber auch nicht ein Nichts.» («Ce n'est pas un quelque chose, mais pas non plus un rien $^{23}$ !»)

4. Si certaines choses sont des instruments (au sens ordinaire), les autres n'en sont pas. Néanmoins toutes les choses peuvent être appelées instruments au sens large du mot, c'est-àdire au sens qu'elles peuvent toutes être employées d'une certaine façon.

Chez Heidegger, le rôle d'une telle notion universelle d'instrument joue la notion de Zeug. De même que chez Wittgenstein la règle n'est pas séparable de ses usages, chez Heidegger (et chez Wittgenstein) l'instrument n'est pas séparable de ses usages. C'est dans l'usage que l'instrument devient ce qu'il est.

«Ein Zeug „,ist“" strenggenommen nie. Zum Sein von Zeug gehört je immer ein Zeugganzes, darin es dieses Zeug sein kann, das es ist. Zeug ist wesenhaft „etwas, um zu...“ (...). In der Struktur „Um-zu“ liegt eine Verweisung von etwas auf etwas ${ }^{24}$.» («Un outil, en toute rigueur, cela n'existe pas. À l'être de l'outil appartient toujours un

\footnotetext{
${ }^{21}$ Heidegger Martin, Sein und Zeit, § 15, p. 69.

${ }^{22}$ Heidegger Martin, Etre et temps, p.74.

${ }^{23}$ Wittgenstein Ludwig, Philosophical Investigations, $§ 304$. Wittgenstein Ludwig, Tractatus logicophilosophiques suivi d'investigations philosophiques, § 304.

${ }^{24}$ Heidegger Martin, Sein und Zeit, p.68.
} 
complexe d'outils au sein duquel il peut être cet outil qu'il est. L'outil est essentiellement , quelque chose pour... “. (...) Dans la structure du ,pour... “ est contenu un renvoi de quelque chose à quelque chose $\left.\mathrm{e}^{25} . »\right)$

Le signe est un exemple de l'instrument/outil au sens large (voir la note 15 ci-dessus). Le signe employé (et non pas isolé), c'est-à-dire, le symbole, montre quelque chose et, ainsi, renvoie à ce qu'il montre.

À la différence de Wittgenstein, Heidegger essaie de mettre à jour la structure générale du renvoyer de l'outil à son usage (du signe à ce qu'il montre), qui indique la localisation matérielle (le fondement) de la signification.

En particulier, pour lui :

«(...) das Zeichensein für... kann selbst zu einer universalen Beziehungsart formalisiert werden, so daß die Zeichenstruktur selbst einen ontologischen Leitfaden abgibt für eine »Charakteristik « alles Seienden überhaupt ${ }^{26} . »(«(\ldots)$ L'être-signe pour..., [lequel] peut lui-même être formalisé en un mode universel de relation, de telle sorte que la structure de signe fournit elle-même un fil conducteur ontologique pour une „,caractérisation“ de tout étant en général $\left.{ }^{27} . »\right)$

C'est en cela que Benoist voit la différence principale entre les approches de Wittgenstein et Heidegger.

Bien évidement, Wittgenstein n'aurait pas posé la question sur le fondement métaphysique de la signification (et du comprendre) comme telle, - la question que pose, et à sa façon résout Heidegger. Néanmoins, il nous semble que la relation entre les deux philosophes est plus étroite que Benoist ne le pense. Par exemple, l'affirmation que tous les jeux de langage ont quelque chose en commun ne contredit la philosophie de Wittgenstein qu'à première vue.

En particulier, on peut être d'accord avec James Bridges qui énonce qu'il y a quelque chose de commun à tous les jeux ${ }^{28}$. Ce qui est commun est ce qu'ils sont tous des jeux. Nous dirions que ce qui est commun chez eux ce n'est pas une propriété (pour ainsi dire explicite) commune, mais une règle (en générale implicite) commune «jeu» (la règle wittgensteinienne, associée avec le mot «jeu »).

Da façon analogue, ce qui est commun à tous les jeux de langage c'est qu'ils sont des pratiques régies par des règles. Dans ce sens, on peut parler d'un fondement métaphysique de la signification et du comprendre.

Le «fil conducteur ontologique » dont parle Heidegger est la règle wittgensteinienne dans son usage.

5. L'analyse wittgensteinienne ne se réduit pas à une analyse thérapeutique grammaticale (c'est-à-dire, conceptuelle) sur laquelle Jocelyn Benoist porte son attention en opposant l'approche de Wittgenstein à celle de Heidegger. De nombreux exemples wittgensteiniens,

\footnotetext{
${ }^{25}$ Heidegger Martin, Être et temps, p. 73.

${ }^{26}$ Heidegger Martin, Sein und Zeit, p. 77.

${ }^{27}$ Heidegger Martin, Être et temps, p. 80.

${ }^{28}$ Bridges Jason, «Wittgenstein's contextualism », in Arif Ahmed (ed.), Wittgenstein's Philosophical Investigations. A Critical Guide, Cambridge UP, 2010.
} 
son analyse de la notion de jeu de langage et du problème « suivre une/la règle » donnent une idée de la nature et de la structure des jeux de langage (usages) à la fois naturels et normatifs (spontanés) - c'est-à-dire, des pratiques naturelles et normatives - , qui jouent le rôle du Donné premier.

Les caractéristiques du jeu de langage comme tel rappellent celles du Dasein heideggérien. (Voir aussi une interprétation analytique du Dasein de Robert Brandom. ${ }^{29}$ ) L'essence du Dasein est dans son existence. L'essence du jeu de langage réside en ce qu'il est l'application de la règle. Dans les deux cas, l'existentia a une priorité par rapport à l'essentia. Le Dasein comme le jeu de langage sont les miens (la mienneté (die Jemeinigkeit) du Dasein). Dans la vie quotidienne, les deux ont le caractère de moyenneté (Durchschnittlichkeit). ${ }^{30}$ Et ainsi de suite.

Chez Wittgenstein, une règle ou un jeu de langage peut être projeté dans un nouveau contexte. Cela donne naissance à un nouveau jeu de langage - un nouvel usage et, respectivement, un nouveau sens authentique, correspondant à une nouvelle (authentique) existence (être). Un jeu de langage a certaines possibilités réelles (pas seulement purement logiques) pour sa projection dans un nouveau contexte, pour son développement et généralisation.

Chez Heidegger, les possibilités potentielles du Dasein, dans lesquelles il s'ouvre, appartiennent à son existence même. Les choses dans le monde se découvrent et sont comprises conformément aux possibilités du Dasein et simultanément avec l'ouverture de son existence. Et quand les choses sont comprises, nous disons qu'elles ont un sens. ${ }^{31}$

Pour Wittgenstein, la signification est l'usage (jeu de langage) ou sa règle (règle du jeu de langage $)^{32}$. Pour Heidegger ${ }^{33}:$ «Sinn ist ein Existenzial des Daseins, nicht eine Eigenschaft, die am Seienden haftet, »hinter« ihm liegt oder als »Zwischenreich« irgendwo schwebt.» («Le sens est un existential du Dasein, non pas une propriété qui s'attache à l'étant, est „derrière“ lui ou flotte quelque part comme ,règne intermédiaire“.$\left.{ }^{34} »\right)$

Dans le $\S 32$ Heidegger explique sa vision du concept de la signification/sens ainsi ${ }^{35}$ :

«Verstanden aber ist, streng genommen, nicht der Sinn, sondern das Seiende, bzw. das Sein. Sinn ist das, worin sich Verständlichkeit von etwas hält. Was im verstehenden Erschließen artikulierbar ist, nennen wir Sinn. Der Begriff des Sinnes umfaßt das formale Gerüst dessen, was notwendig zu dem gehört, was verstehende Auslegung artikuliert. Sinn ist das durch Vorhabe, Vorsicht und Vorgriff strukturierte Woraufhin des Entwurfs, aus dem her etwas als etwas verständlich wird.» («Cependant, ce qui est compris, ce n'est pas en toute rigueur le sens, mais l'étant — ou l'être. Le sens est ce en quoi la compréhensibilité de quelque chose se tient. Ce qui est

\footnotetext{
${ }^{29}$ Brandom Robert, Between Saying and Doing, Oxford UP, 2008.

${ }^{30}$ Heidegger Martin, Sein und Zeit, § 9.

31 Heidegger utilise le mot «Sinn» qu'on traduit en Français comme «sens». Dans ce papier nous ne distinguons pas entre le «sens » et la « signification ». Voir aussi ci-dessous.

${ }^{32}$ Nous ne partageons pas l'avis de Benoist que chez Wittgenstein le slogan « la signification est l'usage » ne se rapporte qu'à l'usage de certains mots, tandis que chez Heidegger il revêt un caractère universel, a un caractère universel.

${ }^{33}$ Heidegger Martin, Sein und Zeit, p. 151.

${ }^{34}$ Heidegger Martin, Être et temps, p. 132.

${ }^{35}$ Heidegger Martin, Sein und Zeit, p. 151.
} 
articulable dans l'ouvrir compréhensif, nous l'appelons le sens. Le concept de sens embrasse la structure formelle de ce qui appartient nécessairement à ce que l'explicitation compréhensive articule. Le sens est le versquoi, tel que structuré par la pré-acquisition, la pré-vision et l'anti-cipation, du projet à partir duquel quelque chose devient compréhensible comme quelque $\left.\operatorname{chose}^{36} . »\right)$

Et ce qui appartient nécessairement à ce que l'explicitation/interprétation compréhensive articule est la règle. C'est la signification au sens de la signification de la règle. Tout ce que l'interprétation/explicitation articule est la signification au sens de la signification de l'usage de la règle. (Voir aussi la note 5 en bas de page.)

Pour les deux philosophes, la dimension réflexive au sens large (en particulier, la réflexion proprement dite ou «interprétation » du texte) est secondaire. Ce qui est primaire, c'est le comprendre (et pour Heidegger le Dasein lui-même est le comprendre du monde à travers sa découverte). L'interprétation/réflexion est une explicitation de ce qui est implicite, et non pas une condition du comprendre (c'est-à-dire qu'elle n'est pas prédéterminée. Donc malgré son objectivité elle ne peut pas être univoque). Elle est aussi le comprendre, seulement plus explicite. «Die Ausbildung des Verstehens nennen wir Auslegung. (...) In der Auslegung wird das Verstehen nicht etwas anderes, sondern es selbst ${ }^{37} . »$ («Cette configuration du comprendre, nous la nommons l'explicitation. (...) Dans l'explicitation, le comprendre ne devient pas quelque chose d'autre, mais lui-même $\left.{ }^{38} . »\right)$

La distinction entre l'explicite et l'implicite joue un rôle fondamental chez Wittgenstein ainsi que chez Heidegger ${ }^{39}$.

Chez Wittgenstein, un nouveau jeu de langage nait comme un nouvel usage de la règle qui est utilisée «spontanément» (dans la partie 2 des Investigations philosophiques Wittgenstein dit que le nouveau (il emploie aussi les termes «spontané » et «spécifique ») est toujours un jeu de langage) et instinctivement, ou « aveuglement ». Mais cet «aveuglement » n'est pas celui qui est rejeté par Heidegger dans l'usage (pratique) de l'instrument ; c'est, au contraire, cette façon particulière du voir pratique dont il parle. Chez Heidegger le Dasein le plus fondamental est instinctif.

6. Chez Wittgenstein, « voir-comme » est un exemple de l'usage de la règle, ou celui du jeu de langage. Ce n'est pas une façon spécifique de voir, mais un aspect d'une image jouant le rôle de règle (ou de super-règle). Une règle est implicite dans le jeu de langage «voircomme », et elle peut être employée autrement.

Une certaine image peut avoir des aspects différents dans le sens qu'elle peut être vue différemment dans des divers contextes. Le passage d'un contexte à un autre s'accompagne d'un changement de l'aspect; c'est-à-dire que l'on voit l'un comme l'autre et vice versa.

\footnotetext{
${ }^{36}$ Heidegger Martin, Être et temps, p. 132.

${ }^{37}$ Heidegger Martin, Sein und Zeit, p. 148.

${ }^{38}$ Heidegger Martin, Être et temps, p. 130.

${ }^{39}$ Encore une fois: chez Wittgenstein avant tout il s'agit du langage, tandis que chez Heidegger - du monde. Pour Heidegger, l'explicitation/interprétation elle-même est un usage pratique du monde. Des exemples sont «Zubereiten, Zurechtlegen, Instandsetzen, Verbessern, Ergänzen» (accommoder, préparer, réparer, améliorer, compléter) (Heidegger Martin, Sein und Zeit, pp. 148-149. Heidegger Martin, Etre et temps, p. 130).
} 
L'image elle-même reste neutre par rapport au choix du contexte, et joue le rôle de (super)règle explicite.

Si cette image est considérée sans aucun rapport avec ses possibles usages elle devient dénuée de sens. Un processus structuré prédéterminé permettant d'obtenir les usages d'une telle image abstraite et décontextualisée n'existe pas. «The man who says "surely, these are two different usages" has already decided to use a two-way schema, and what he said expressed this decision ${ }^{40} . »$ («L'homme qui dit „bien sur, il existe deux usages possibles“, a déjà pris la décision d'utiliser un schéma à deux significations, et ce qu'il a dit a exprimé cette décision.» Notre traduction.)

La signification est l'usage. Nous interprétons ce slogan au sens le plus large, admettant par exemple l'existence de concepts phénoménaux (donc de la signification phénoménale comme l'usage phénoménal). Notamment, on peut parler non seulement de la signification du voircomme (où « comme » est complètement explicite) comme l'usage correspondant, mais aussi de la signification phénoménale du voir-comme (où «comme » est entièrement ou en partie implicite) comme l'usage phénoménal correspondant.

Et inversement: l'usage est la signification. Seulement quand on commence a prêter attention aux détails particuliers de l'usage, il commence à se détacher de la signification correspondante, et l'identité «la signification est l'usage», initialement signifiant la dépendance de la signification du contexte, sa variabilité (il est impossible de comprendre la notion de la signification en séparation de la notion de l'usage et vice versa), commence à se transformer en identité entre la signification (l'usage d'un concept) et l'usage (l'usage d'un autre concept) - c'est-à-dire une autre signification, - renvoyant au seul et même référent.

Une règle générale, un concept (général ou concret) ou une description (plus ou moins concrète) dit «synoptique ${ }^{41}$ peuvent être établis comme le résultat d'une explicitation d'une expérience perceptive instinctive voir-comme. L'explicitation/interprétation transforme cette dernière dans l'expérience du voir un objet. Dans un autre sens, une «interprétation» est une autre «voir-comme» (c'est-a-dire «voir autrement») - un autre aspect de la règle ${ }^{42}$. Le passage d'un aspect à un autre signifie voir l'un comme l'autre. Ce comme est l'air de famille. Elle s'établit grâce à la règle commune qui appartient à tous les aspects.

\footnotetext{
${ }^{40}$ Wittgenstein Ludwig, The Blue Book.

41 Selon nous, la notion Wittgensteinienne de la description (vision) synoptique correspond à la notion heideggérienne de circonspection (Umsicht). «Die Umsicht entdeckt, das bedeutet, die schon verstandene »Welt « wird ausgelegt. Das Zuhandene kommt ausdrücklich in die verstehende Sicht.» (Heidegger Martin, Sein und Zeit, p. 148.) («La circon-spection découvre, ce qui veut dire que le „monde“ déjà compris est explicité. L'à-portée-de-la-main vient expressément à la vue compréhensive.» (Heidegger Martin, Être et temps, p. 130.)) ${ }^{42} \mathrm{C}$ 'est dans ce sens-là qu'on peut interpréter chez Heidegger l'«interprétation/explicitation» comme la formation (sich auszubilden) du comprendre (ou «projection du comprendre») (voir tout au début du $§ 32$ Sein und Zeit).
}

Pour Heidegger «Das Dasein entwirft als Verstehen sein Sein auf Möglichkeiten. Dieses verstehende Sein zu Möglichkeiten ist selbst durch den Rückschlag dieser als erschlossener in das Dasein ein Seinkönnen.» (Heidegger Martin, Sein und Zeit, p. 148.) («En tant que comprendre, le Dasein projette son être vers des possibilités. Cet être compréhensif pour des possibilités est lui-même, par le rejaillissement de celle-ci en tant qu'ouvertes vers le Dasein, un pouvoir-être.» Heidegger Martin, Être et temps, p. 130.) (Comparons cela avec la métaphore wittgensteinienne du lit de la rivière qui se rapporte à la règle (la rivière joue le rôle des applications de la règle). Les nouvelles applications d'une règle affectent la règle elle-même et entrainent un changement graduel.) Ainsi, le projeter du comprendre comporte la possibilité de sa «formation »/interprétation. (Voir le début du $\$ 32$ Sein und Zeit.) 
Une description du voir-comme en termes de la règle et ses usages peut être appelée description de la «structure » de ce jeu de langage (chez Wittgenstein cette description est implicite et non pas explicite). Elle est analogue à la description proposée par Heidegger: «Das »Als« macht die Struktur der Ausdrücklichkeit eines Verstandenen aus; es konstituiert die Auslegung ${ }^{43}$.» (Le « comme » constitue la structure de l'expressivité de ce qui est compris ; il constitue l'explicitation ${ }^{44}$.)

Voici ce que Heidegger écrit de l'expérience phénoménale du voir-comme:

«Daß im schlichten Hinsehen die Ausdrücklichkeit eines Aussagens fehlen kann, berechtigt nicht dazu, diesem schlichten Sehen jede artikulierende Auslegung, mithin die Als-struktur abzusprechen. Das schlichte Sehen der nächsten Dinge im Zutun-haben mit... trägt die Auslegungsstruktur so ursprünglich in sich, daß gerade ein gleichsam als-freies Erfassen von etwas einer gewissen Umstellung bedarf. Das Nur-noch-vor-sich-Haben von etwas liegt vor im reinen Anstarren als Nicht-mehr-verstehen. Dieses als-freie Erfassen ist eine Privation des schlicht verstehen den Sehens, nicht ursprünglicher als dieses, sondern abgeleitet aus ihm. Die ontische Unausgesprochenheit des »als« darf nicht dazu verführen, es als apriorische existenziale Verfassung des Verstehens zu übersehen ${ }^{45}$.» («Que l'expressivité d'un énoncé puisse faire défaut dans l'avisement pur et simple, cela n'autorise pas à dénier à ce pur et simple voir toute explicitation articulante, donc la structure du „comme“. Le voir pur et simple des choses les plus proches dans l'avoir affaire avec... inclut si originairement la structure d'explicitation que la saisie de quelque chose comme-libre, pour ainsi dire, a justement besoin d'une certaine inversion de sens. Dans le pur regard qui fixe, l'avoir-devant-soi-sans-plus-quelque-chose est présent, en tant que ne-plus-comprendre. Cette saisie-comme-libre est une privation du voir purement et simplement compréhensif, elle n'est pas plus originaire que lui, mais en dérive. Le fait ontique que le « comme » ne soit pas exprimé ne doit pas conduire à le méconnaître en tant que constitution existentiale apriorique du comprendre $\left.{ }^{46} . \gg\right)$

Le comprendre primaire est celui d'une règle implicite ou d'un certain de ses usages concrets. Une interprétation est un saisi d'un autre usage de la règle ou une explicitation de son usage initial. D'où la structure «comme ». Dans ce cas l'expérience purement phénoménale (visuelle) précède l'expérience linguistiquement exprimée, contenant le «comme» explicitement.

7. Ainsi nous pensons que l'analogie entre Wittgenstein et Heidegger, notamment, en ce qui concerne la notion d'usage, n'est pas superficielle. Heidegger explicite la métaphysique implicite wittgensteinienne. Le pragmatisme de Wittgenstein peut être théorisé.

Il nous semble que ceci se confirme, par exemple, par les travaux de Robert Brandom ${ }^{47}$. Notamment, son pragmatisme analytique ${ }^{48}$ s'inspire par les idées du second Wittgenstein (bien qu'a notre avis Brandom n'interprète pas toujours correctement Wittgenstein ${ }^{49}$ ).

\footnotetext{
${ }^{43}$ Heidegger Martin, Sein und Zeit, p.149.

${ }^{44}$ Heidegger Martin, Être et temps, p. 131.

${ }^{45}$ Heidegger Martin, Sein und Zeit, p. 149.

${ }^{46}$ Heidegger Martin, Être et temps, p. 131.

${ }^{47}$ Brandom Robert, Between Saying and Doing, Oxford UP, 2008. Brandom Robert, Reason in Philosophy. Animating Ideas, Harvard UP, 2009. Brandom Robert, Perspectives on Pragmatism, Harvard UP, 2011.

${ }^{48}$ Brandom Robert, Between Saying and Doing, Oxford UP, 2008.

${ }^{49}$ Nous partageons, en grande partie, la critique de la vision de Brandom de la philosophie de Wittgenstein, proposée par John McDowell.
} 


\section{Références}

Benoist Jocelyn, «Le mythe de l'usage », Les Etudes philosophiques, 2010, 3, n 4, pp. 417432

Benoist Jocelyn, Concepts, Paris, Les éditions CERF, 2010/2011.

Benoist Jocelyn, Eléments de philosophie réaliste, Paris, Vrin, 2011.

Benoist Jocelyn, « Making Ontology Sensitive », Cont. Philos. Rev., 2012, 45, pp. 411-424.

Brandom Robert, Tales of the Mighty Dead, Cambridge: Harvard, 2002

Brandom Robert, Between Saying and Doing, Oxford UP, 2008.

Brandom Robert, Reason in Philosophy. Animating Ideas, Harvard UP, 2009

Brandom Robert, Perspectives on Pragmatism, Harvard UP, 2011

Bridges Jason, «Wittgenstein's contextualism », in Arif Ahmed (ed.), Wittgenstein's Philosophical Investigations. A Critical Guide, Cambridge UP, 2010.

Haugeland John, « Reading Brandom Reading Heidegger», European Journal of Philosophy, 2005, 13:3, pp. 421-428.

Heidegger Martin, Sein und Zeit (1926), Tübingen, Max Niemeyer, 1967. (Accessible sur Internet.)

Heidegger Martin, Sein und Zeit (1926), Tübingen, Max Niemeyer Verlag, 2006.

Heidegger Martin, Etre et temps, tr. fr. Emmanuel Martineau, Edition numérique HorsCommerce, 1985.

Rouse Joseph, How Scientific Practices Matter, Chicago, 2002.

Rouse Joseph (ed.), John Heugeland, Dasein Disclosed: John Haugeland's Heidegger, Harvard University Press, 2013.

Wittgenstein Ludwig, Tractatus logico-philosophiques suivi d'investigations philosophiques, tr. fr. Pierre Klossowski, Gallimard, 1961.

Wittgenstein Ludwig, Philosophical Investigations (1953), Blackwell Publishing, 2001.

Wittgenstein Ludwig, Recherches philosophiques, trad. fr. Françoise Dastur, Maurice Élie, Jean-Luc Gautero, Dominique Janicaud, Élisabeth Rigal, Gallimard, 2005.

Wittgenstein Ludwig, The Blue Book, Oxford, Blackwell, 1958. 